\title{
The role of the TLR4 signaling pathway in cognitive deficits following surgery in aged rats
}

\author{
YI WANG, HUIJUAN HE, DAN LI, WENJING ZHU, KAIMING DUAN, YUAN LE, YAN LIAO and YANGWEN OU \\ Department of Anesthesiology, The Third-Xiangya Hospital, Central South University, Changsha, Hunan 410013, P.R. China
}

Received November 24, 2012; Accepted January 31, 2013

DOI: $10.3892 / \mathrm{mmr} .2013 .1322$

\begin{abstract}
Postoperative cognitive dysfunction (POCD), common in elderly patients, refers to a decline in cognitive function following surgery, which may persist or even evolve into Alzheimer's disease (AD). Despite great efforts, the mechanism of POCD remains unclear. In the present study, we tested the hypothesis that Toll-like receptor 4 (TLR4) on microglia contributes to POCD. Shortly after surgery, aged rats demonstrated significant deficits in memory and learning, accompanied by the activation of microglia, marked upregulation of TLR4 on microglia in the hippocampus, as well as an increased expression of two downstream factors [myeloid differentiation factor 88 (MyD88) and TIR-domain-containing adapter-inducing interferon- $\beta$ (TRIF)] and pro-inflammatory cytokines [including tumor necrosis factor $\alpha(\mathrm{TNF}-\alpha)$ and interleukin $1 \beta$ (IL-1 $\beta$ )]. With an increase in time following surgery, the expression of TLR 4 and the aforementioned factors and pro-inflammatory cytokines gradually returned to normal, as did the cognitive function of the aged rats. In conclusion, our study suggests that the activation of TLR4 signaling on microglia may act as an underlying mechanism of POCD.
\end{abstract}

\section{Introduction}

Postoperative cognitive dysfunction (POCD) refers to a decline in cognitive function following surgery, characterized by an impairment of memory, concentration and comprehension, and a decreased ability to process information $(1,2)$. The underlying molecular mechanism of POCD has not been fully identified; however, accumulating hypotheses have been proposed based on experimental evidence indicating that anesthetics and surgery may act as potential causes for POCD (3). As neuroinflammation has been demonstrated to be associated with cognitive defects in many central nervous system (CNS) diseases, it may also play a crucial role in POCD (4). It has

Correspondence to: Professor Yangwen Ou, Department of Anesthesiology, The Third-Xiangya Hospital, Central South University, 138 Tongzipo Road, Changsha, Hunan 410013, P.R. China E-mail: ouyangwen2010@yahoo.cn

Key words: POCD, microglia, TLR4, neuroinflammation, aged been hypothesized that anesthetics lead to neuroinflammation through upregulation of the release of pro-inflammatory factors, such as tumor necrosis factor $\alpha$ (TNF- $\alpha)$ and interleukin $1 \beta$ (IL-1 $\beta$ ) $(5,6)$. Furthermore, as surgery has been demonstrated to cause a profound systemic inflammatory response, in an approximate association with the magnitude of tissue damage, it may also act as a key factor in POCD (7).

Peripheric pro-inflammatory cytokines may enter the CNS through deficient or damaged areas of the blood-brain barrier (BBB) and subsequently trigger a series of neuroinflammation over the entire surface of the brain (8). As resident immunocytes, microglia rapidly alter the expression of various molecules in response to changes in the brain, to maintain the balance of the internal environment. Once activated, microglia highly express masses of receptors, subsequently initiating various signaling pathways and eventually releasing numerous pro-inflammtory factors (9). Toll-like receptor 4 (TLR4), a type of pattern recognition receptor (PRR) mainly expressed on microglia, may be activated by numerous endogenous and exogenous factors, and is involved in innate and adaptive immunity by producing numerous cytokines and pro-inflammatory factors via myeloid differentiation factor 88 (MyD88)-dependent and MyD88-independent pathways $(10,11)$. As accumulating evidence has demonstrated that the activation of TLR4 leads to neuroinflammation, we hypothesized that TLR4 may contribute to POCD through the induction of neuroinflammation.

In the present study, to evaluate whether TLR4 was associated with POCD, we investigated the cognitive functions of aged rats following surgery, as well as the activation of microglia and their expression of TLR4. We also examined certain key downstream factors and compared their changes before and after surgery.

\section{Materials and methods}

Animals and groups. Sixty Sprague-Dawley female rats (22-23 months old, weighing 450-550 g) were purchased from the Dongchuang Laboratory Animal Centre (Hunan, China) and fed separately in a light-, temperature- and humidity-controlled environment with free access to food and water.

Experiments were conducted in accordance with the guidelines for care and use of laboratory animals of the Ethics Committee of Central South University (Hunan, China). The 
rats were randomly assigned to four groups. In the control group (group $\mathrm{C} ; \mathrm{n}=15$ ), the rats did not undergo surgery. The remaining 45 rats were divided into 3 parallel operation groups (groups S1, S3 and S7; n=15, respectively). All the rats were trained in the Morris water maze (MWM) for 6 days. Subsequently, rats in the operation groups underwent splenectomy under isoflurane anesthesia, and were tested by the MWM again on postoperative days 1,3 and 7, respectively.

Tissue treatment. The experimental animals were sacrificed under anesthesia, following the spatial working tests. Hippocampal tissues of 8 rats in each group were quickly separated and stored in liquid nitrogen prior to use. The remaining animals in each group were perfused transcardially with cold physiological saline followed by cold $4 \%$ paraformaldehyde solution in 0.1 M PBS. Then, the brain tissue was dissected and stored in $1 \%$ sodium azide in double distilled water (DDW), and subsequently embedded in optimum cutting temperature (OCT) tissue freezing medium and sectioned for immunofluorescence.

$M W M$. The rats were trained with the platform in a fixed location for three trials per day, for six consecutive days. The subjects were placed on the platform $30 \mathrm{sec}$ prior to the start of each trial, and released into the water from one of three randomly assigned release points (NW, SW and NE). The platform was located in the SE. In each trial, one rat was allowed to swim until it landed on the platform. If a rat failed to find the platform within $60 \mathrm{sec}$, it was picked up and placed on the platform for $15 \mathrm{sec}$. The animal was maintained on the platform for $30 \mathrm{sec}$ between trials. The rats in groups S1, S3 and S7 underwent surgery on the seventh day. On postoperative days 1, 3 and 7, rats were subjected to a reversal test, in which the platform was relocated to the opposite quadrant of the pool. The reversal learning revealed whether the animals were able to extinguish their initial learning of the platform's position and acquire a direct path to the new goal position. Swimming distance, speed and latency to the platform were recorded by video tracking mounted on the ceiling, and digital images were analyzed by water maze software (Smart Junior Software, Panlab, Barcelona, Spain).

Immunofluorescence. Sections were blocked in 3\% normal goat serum at room temperature for $1 \mathrm{~h}$, and then incubated with rabbit anti-rat TLR4 antibody (1:100; Abbiotec, LLC, San Diego, CA, USA) antibody at $4^{\circ} \mathrm{C}$ overnight. Following washing wih PBS, sections were incubated with biotinylated goat anti-rabbit (TLR4) or horse anti-rabbit (ox-42) IgG secondary antibody at $37^{\circ} \mathrm{C}$ for $2 \mathrm{~h}$, followed by reaction with red fluorescein for $2 \mathrm{~h}$ after washing. Prior to blocking in $3 \%$ normal horse serum at room temperature for $1 \mathrm{~h}$ again, washing was carried out. Incubation with mouse anti-rat ox-42 antibody (1:50; Millipore, Billerica, MA, USA) was carried out at $4^{\circ} \mathrm{C}$ overnight. Subsequently, biotinylated horse anti-rabbit IgG secondary antibody and green fluorescein were applied successively for $2 \mathrm{~h}$ for each antibody, and washing was conducted prior to each step. Sections were observed through a fluorescence microscope equipped with a digital camera.

Protein extraction and western blot analysis. Total RNA was extracted from homogenization of $200 \mathrm{mg}$ hippocampus tissue
Table I. Primer sequences.

\begin{tabular}{ll}
\hline Primer (length) & Sequence \\
\hline TLR4 (523bp) & 5'-3': GAAGCCTCGTGCTCCCTGGC \\
& 3'-5': GAAGCCTCGTGCTCCCTGGC \\
MyD88 (163bp) & 5'-3': GCCGGAGCTTTTCGACGCCT \\
& 3'-5': GAGCTCGCTGGCGATGGACC \\
TRIF (273bp) & 5'-3': CTGATGCTCACCTGCGGCCA \\
& 3'-5': CAGCCGGGCATCCTTGCACT \\
TNF- $\alpha$ (161bp) & 5'-3': TGACCCCCATTACTCTGACC \\
& 3'-5': GGCCACTACTTCAGCGTCTC \\
IL-1 3 (245bp) & 5'-3': CTCCATGAGCTTTGTACAAGG \\
& 3'-5': TGCTGATGTACCAGTTGGGG
\end{tabular}

TLR4, Toll-like receptor 4; MyD88, myeloid differentiation factor 88; TRIF, TIR-domain-containing adapter-inducing interferon- $\beta$; TNF- $\alpha$, tumor necrosis factor $\alpha$; IL-1 $1 \beta$, interleukin $1 \beta$.

samples using cold RIPA lysis buffer. The protein concentrations were measured by a bicichoninic acid (BCA) protein assay kit (Pierce Biotechnology, Inc., Rockford, IL, USA). The samples $(20 \mu \mathrm{g})$ were loaded and separated by SDS-PAGE and then electrophoretically transferred to PVDF membranes (Pall Corporation, Port Washington, NY, USA). The membranes were probed with various antibodies, including TLR4, MyD88, TIR-domain-containing adapter-inducing interferon- $\beta$ (TRIF), TNF- $\alpha$ and IL-1 $\beta$, and visualized with peroxidase and an enhanced chemiluminescence system (Pierce Biotechnology, Inc.). The concentrations of primary antibodies are as follows: Rabbit anti-TLR4 (1:500; Abbiotec LLC), rabbit anti-MyD88 $(1: 1,000)$, rabbit anti-TRIF $(1: 1,000)$, rabbit anti- IL-1 $\beta$ (1:1000) and rabbit anti-TNF- $\alpha$ (1:800; Cell Signaling Technology, Inc., Beverly, MA, USA).

RNA extraction and reverse transcription-polymerase chain reaction $(R T-P C R)$. Total RNA was extracted from homogenization of $200 \mathrm{mg}$ hippocampus tissue samples using TRIzol reagent (Invitrogen, Life Technologies, Carlsbad, CA, USA) and RT-PCR was then performed. Briefly, synthesis of the first strand of complementary DNA was conducted using the RNA PCR kit (AMV) Version 3.0. RT-PCR was performed at $30^{\circ} \mathrm{C}$ for $10 \mathrm{~min}, 42^{\circ} \mathrm{C}$ for $30 \mathrm{~min}$ and terminated by heating to $99^{\circ} \mathrm{C}$ for $5 \mathrm{~min}$. TLR4, MyD88, TRIF, TNF- $\alpha$ and IL-1 $\beta$ were then amplified by PCR. Primer sequences and amplification sizes are provided in Table I. $\beta$-actin was used as an internal reference to determine the relative expression levels of mRNA.

Statistical analysis. Statistical analysis was performed using SPSS 17.0 statistical software (SPSS Inc., Chicago, IL, USA). Data from the RT-PCR and western blot analysis were analyzed by a one-way ANOVA, in which age and operation were the dependent variables. A one-way repeated measures ANOVA was used to analyze the training behavioral parameters. A separate one-way ANOVA was performed to analyze the effects of surgery on working memory performance during the reversal testing. A value of 


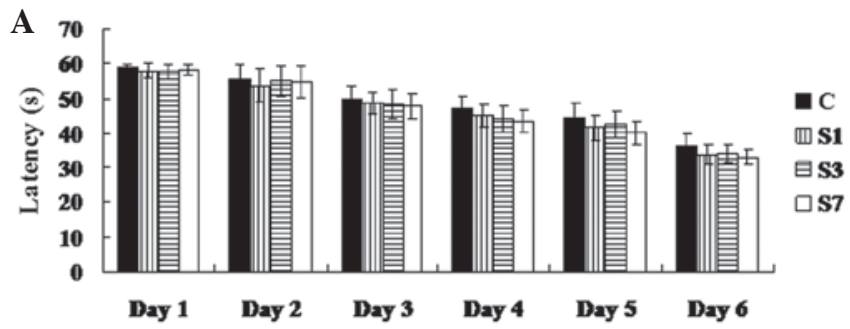

B
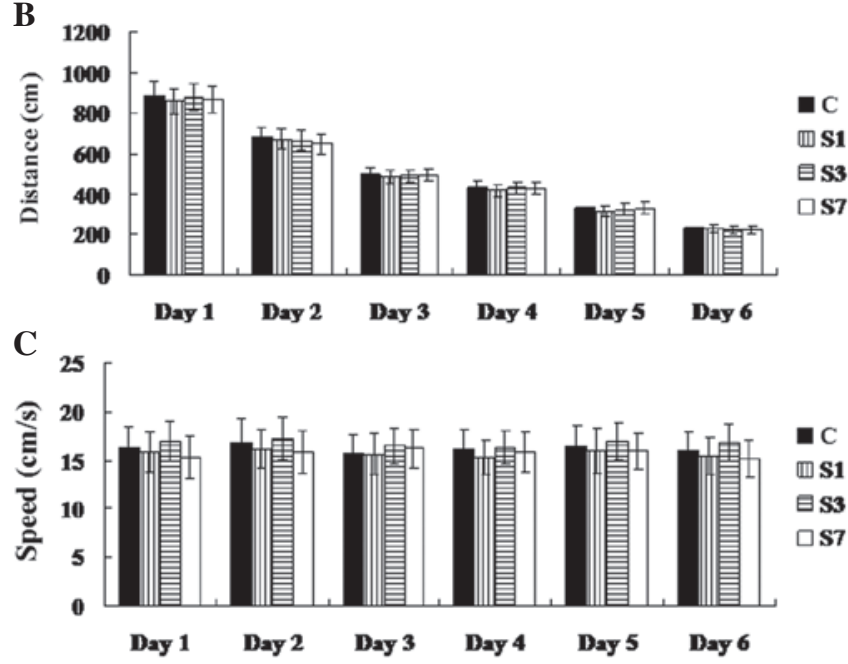

Figure 1. Swimming data of aged rats for six consecutive training days in the Morris water maze. (A) Latency to the platform, (B) swimming distance and (C) swimming speed during training days. Results are presented as the mean \pm standard deviation.

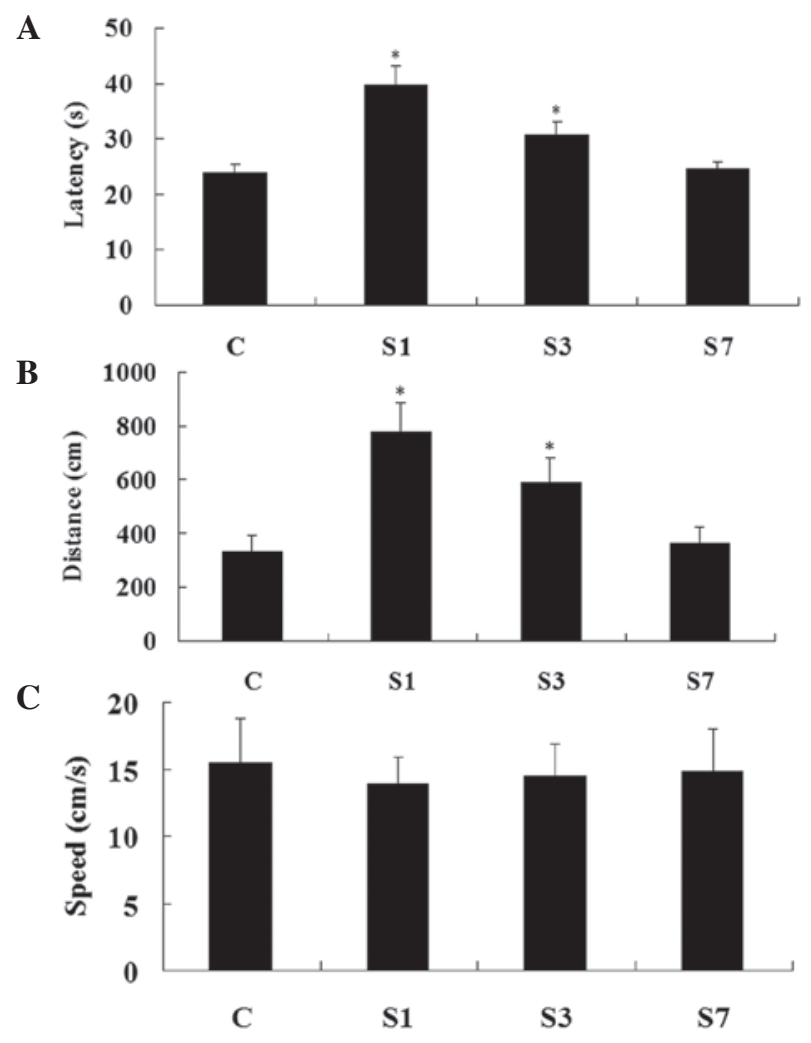

Figure 2. Swimming data of aged rats following splenectomy in reversal learning testing. (A) Latency to the platform, (B) swimming distance and (C) swimming speed, preoperatively and postoperatively. Results are presented as the mean \pm standard deviation. ${ }^{*} \mathrm{P}<0.05$. C, control group; S1, S3 and $\mathrm{S} 7$, operation group on days 1,3 and 7 , postoperatively.

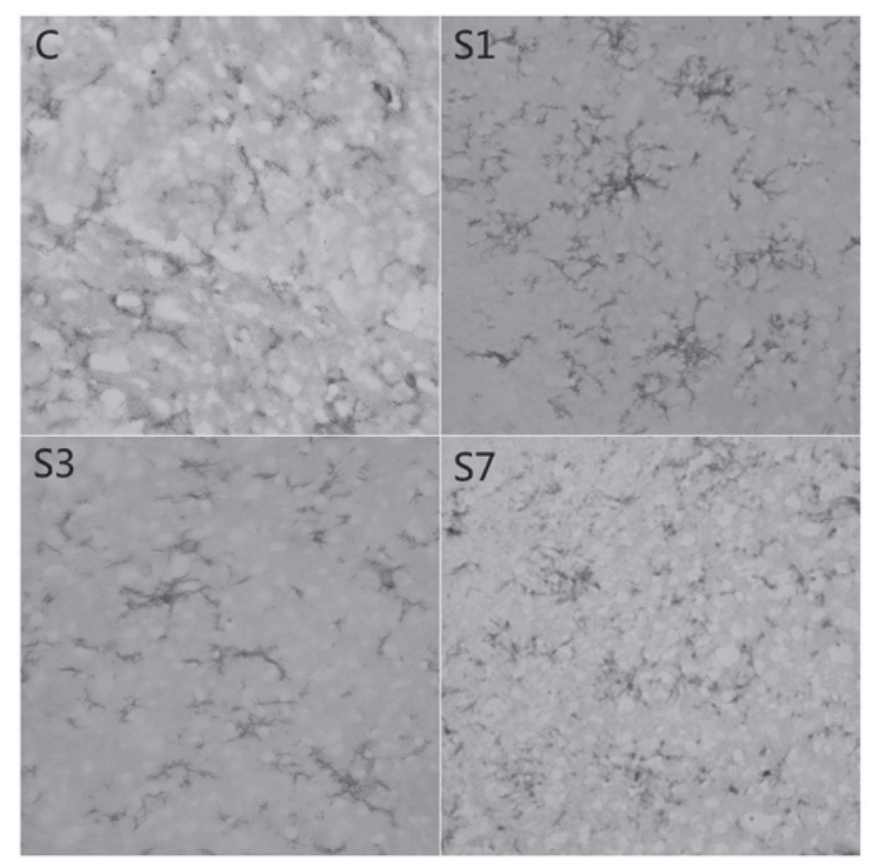

Figure 3. The morphology of the microglia of the aged rats in each group. C, control group; S1, S3 and S7, operation group on days 1, 3 and 7, respectively, postoperatively.

$\mathrm{P}<0.05$ was considered to indicate a statistically significant difference.

\section{Results}

Aged rats showed a transient cognitive deficit in the early days following surgery. Rats in all groups were trained to search the platform in the MWM for 6 days prior to surgery. Latency and distance were applied as two key parameters to evaluate their spatial memory ability. As demonstrated in Fig. 1, latency and distance gradually decreased during the training, suggesting that their spatial memory was built. On the 6th day following training, almost all rats were able to rapidly find the platform in the MWM each time. On postoperative day 1, the distance and latency in group S1 were increased compared with group $\mathrm{C}(\mathrm{P}<0.05)$. On postoperative day 3, although decreased, the distance and latency in group $\mathrm{S} 3$ remained higher than those of group $\mathrm{C}(\mathrm{P}<0.05)$. On postoperative day 7 , these two key parameters returned to normal (compared with group $\mathrm{C}$, $\mathrm{P}>0.05)$. Swimming speeds in these four groups demonstrated no significant difference ( $\mathrm{P}>0.05$ ) (Fig. 2). Accordingly, these data suggest that the rats suffered a transient cognitive deficit following surgery.

Microglia were activated and TLR4 was upregulated in the hippocampus following surgery. As demonstrated in Fig. 3, surgery led to changes in the morphology of the microglia. The expanded body and the long branches are characteristic of activation, indicating that the microglia changed from a resting to an activated state. With an increase in time following surgery, the morphology of the microglia gradually returned to normal, suggesting that the microglia altered from an activated to a resting state. 


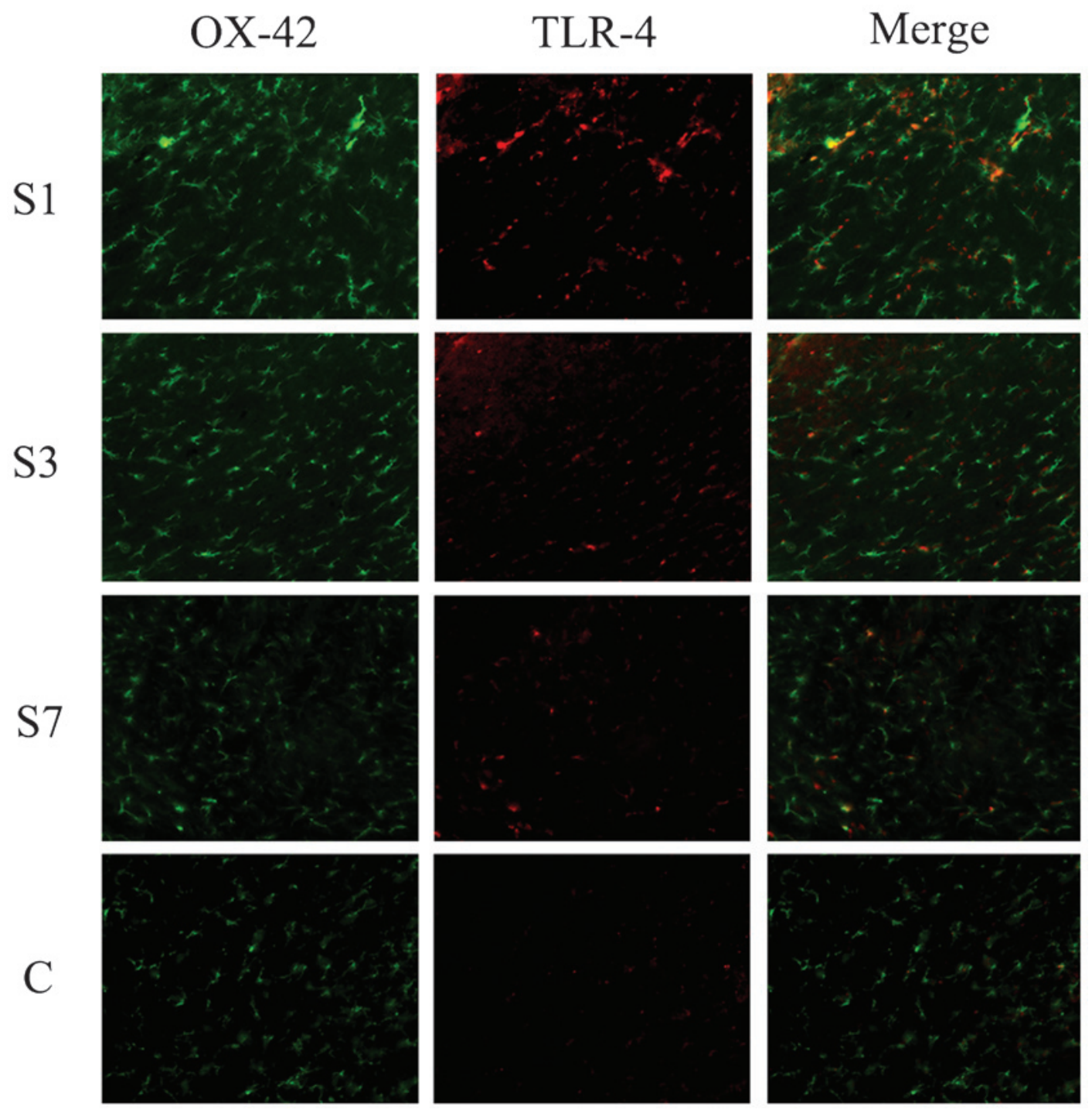

Figure 4. Expression of TLR4 in the microglia. OX-42 was used to show the microglia. C, control group; S1, S3 and S7, operation group on days 1, 3 and 7, respectively, postoperatively.

TLR4 is mainly expressed on microglia in the brain. As demonstrated in Fig. 4, on postoperative day 1, the number of TLR4(+) microglia in group S1 was significantly increased compared with that in the control group $(\mathrm{P}<0.05)$. Subsequently, the expression of TLR4 decreased over time, following surgery. On postoperative day 3, its expression in group S3 remained higher than that of group $\mathrm{C}(\mathrm{P}<0.05)$. In addition, only a few TLR4(+) microglia were detected in the hippocampus on postoperative day 7 , similar to that of group $\mathrm{C}(\mathrm{P}>0.05)$. To further verify these findings, we determined the expression of TLR4 using RT-PCR and western blot analysis, the results of which showed a similar pattern to those of the immunofluorescence experiments (Fig. 5). Based on these data, the expression of TLR4 changed with the behavior, suggesting that TLR4 may be related to the cognitive deficit.

Expression of MyD88 and TRIF in the hippocampus. MyD88 and TRIF are key downstream components of the TLR4 signaling pathway. As demonstrated in Fig. 5, on postoperative day 1 , the expression of MyD88 and TRIF were significantly upregulated in the hippocampus at both the mRNA and protein levels, compared with those in group $\mathrm{C}$ $(\mathrm{P}<0.05)$, suggesting that both TLR4 downstream pathways were activated. Moreover, the expression of MyD88 and TRIF demonstrated a similar decrease to TLR4 over time, following surgery.

Release of pro-inflammatory factors TNF- $\alpha$ and $I L-1 \beta$ in the hippocampus. To investigate the neuroinflammation, we determined two key pro-inflammatory factors in brain, TNF- $\alpha$ and IL-1 $\beta$. Data from the RT-PCR and western blot analysis revealed that the expression levels of TNF- $\alpha$ and IL-1 $\beta$ were increased in the early days following surgery, peaking on postoperative day $1(\mathrm{P}<0.05)$. Subsequently, their expression levels gradually reduced with an increase in time following surgery. On postoperative day 7, the expression of TNF- $\alpha$ returned to normal; however, the expression of IL-1 $\beta$ remained higher than that of group $C$ $(\mathrm{P}<0.05)$ (Fig. 4). According to the data thus far, until postoperative day 7 , neuroinflammation and cognitive deficit had disappeared. 
A
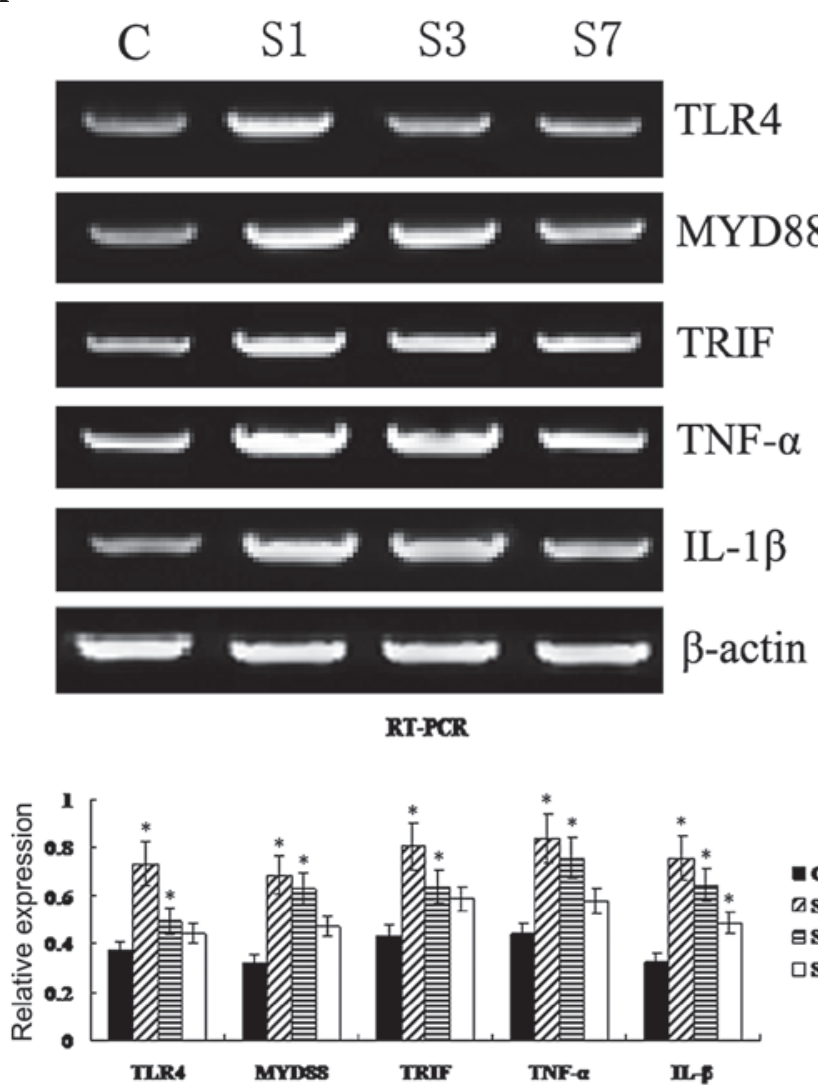

B

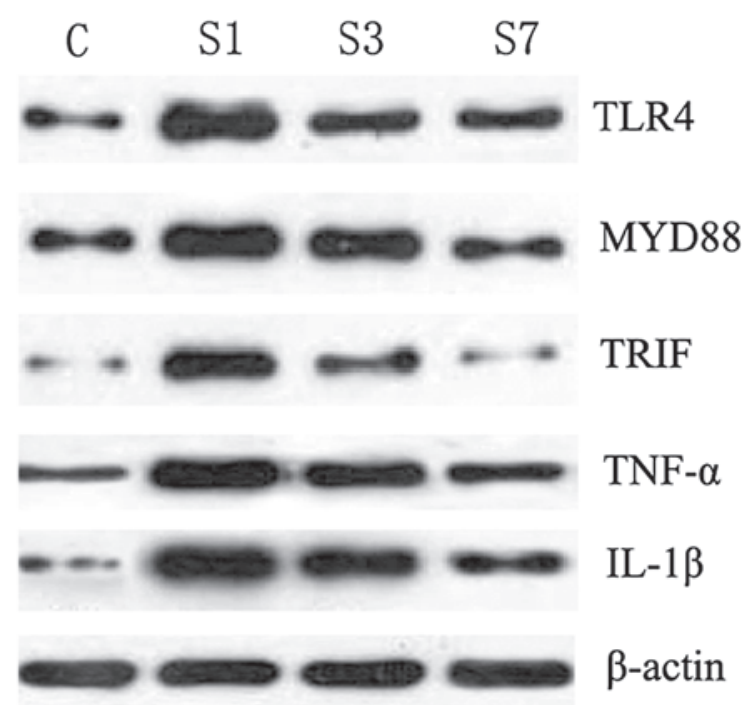

Western blot analysis

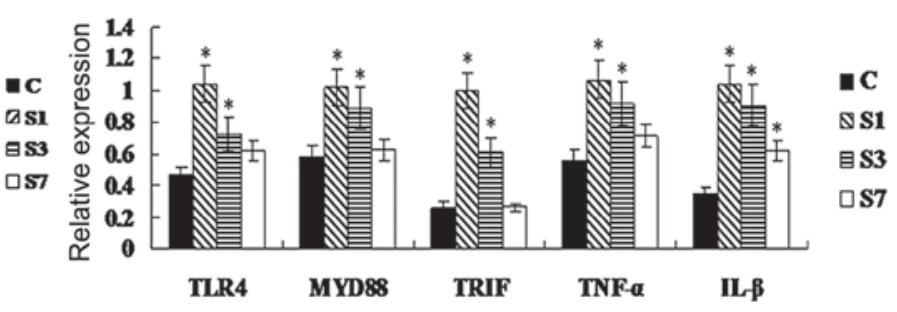

Figure 5. Expression of key factors in the TLR4 signaling pathway. RT-PCR (A) and western blot analysis (B) were used to determine the expression of several key factors in the TLR4 signaling pathway, including TLR4, MyD88, TRIF, TNF- $\alpha$ and IL-1 $\beta$. Results are presented as the mean \pm standard deviation. ${ }^{*}<0.05$. $\mathrm{C}$, control group; S1, S3 and S7, operation group on days 1, 3 and 7, postoperatively.

\section{Discussion}

POCD is common in elderly patients. Its high morbidity is attributed to multiple reasons, including neuroinflammation (12). Accumulating evidence supports the theory that non-infectious neuroinflammation is associated with POCD (7). As a type of resident immune cell, microglia stabilize the microenvironment in the brain. Any changes disturbing homeostasis in the brain lead to the activation of microglia, such as invading pathogens or brain injury (13). Activated microglia protect neurons from damage by phagocytosing pathogens or synthesizing and secreting pro-inflammatory factors (14). However, once the microglia are over-activated, excessive pro-inflammatory factors are produced and released, which leads to an excessive neuroinflammatory response and is harmful to neurons (15). It has been universally accepted that drugs, as well as stress from surgery, are able to disrupt homeostasis of the whole body, including the brain, which leads to systematic inflammation $(1,16)$. Peripheric pro-inflammatory factors are capable of entering BBB-deficient areas and activating the microglia, which leads to the activation of numerous receptors and thus various downstream signaling pathways $(8,11)$.

TLR4 is a type of PRR mainly expressed on the membrane of microglia, responsible for exogenous ligands, such as lipopolysaccharide (LPS), and endogenous ligands, such as HMGB1 and heat-shock protein 70 (HSP 70) $(17,18)$. When peripheric pro-inflammatory factors enter the brain, some of these may activate TLR4 signaling pathways (19). It has been demonstrated that TLR4 signaling pathways include two branches; the MyD88-dependent and TRIF-dependent pathways (20). The activation of these two pathways induces the phosphorylation of IKBs and their subsequent degradation, which then promotes the nuclear translocation of the transcription factor nuclear factor $\kappa \mathrm{B}(\mathrm{NF}-\kappa \mathrm{B})(21)$. Once NF- $\kappa \mathrm{B}$ enters into the nucleus, it promotes the transcription of various target genes, including certain important pro-inflammatory cytokines, such as IL- $1 \beta$ and TNF- $\alpha$ (21).

At present, whether TLR4 protects or damages the neurons remains unclear. Although some evidence suggests that TLR signaling mediates beneficial effects in the CNS, it has also been demonstrated that TLR-induced activation of microglia and the release of pro-inflammatory molecules are responsible for neurotoxic processes in various CNS diseases $(8,22,23)$. Moreover, numerous studies on CNS diseases, cerebral ischemia and injury have suggested that expression of TLR4 is associated with neurodegeneration $(13,24,25)$. As a result, TLR4-activated microglia may injure the neurons by releasing excessive cytokines, leading to memory and learning deficits. In addition, inflammation has been confirmed to contribute to neurodegeneration at the late stage of Alzheimer's disease (AD); however, it may also play a crucial role in the early stage. Recently, POCD has been regarded as the preliminary state of $\mathrm{AD}$, due to their similarity in clinical symptoms and 
pathological changes $(4,26)$. Therefore, a common or similar mechanism may exist between them.

As demonstrated in this study, shortly following surgery, the aged rats showed transient defects in memory and learning; simultaneously, the expression of TLR4 was significantly upregulated. The distinct cognitive deficit was evident on postoperative day 1 , as was the peak in expression of TLR4, IL-1 $\beta$ and TNF- $\alpha$. Subsequently, this cognitive deficit gradually recovered over time, accompanied by the expression of TLR4 and pro-inflammatory cytokines returning to normal. In this study, the neuroinflammation in the aged brain following surgery was consistent with that of previous studies, and was likely to be due to the increased expression of TLR4.

POCD is a global problem, which is becoming worse along with the increased life-expectancy of society (27). Therefore, it is important, for humans, to identify the molecular mechanism of POCD. However, few effective precautions and therapeutic strategies have been well established. Our findings suggest that the activation of TLR4 contributed to postoperative neuroinflammation in the aged brain by activating MyD88- and TRIF-dependent signaling pathways, and ultimately leading to POCD. In summary, the present study identified the crucial role of TLR4 in POCD, and indicated that TLR4 may become a novel target for taking precautions and for therapy.

\section{Acknowledgements}

This study is supported by a grant from the National Natural Science Foundation of China (30871306), the 125 Program of The third-Xiangya Hospital, Central South University and the Science-Technology Foundation of Hunan Province, China (2010sk3111).

\section{References}

1. Coburn M, Fahlenkamp A, Zoremba N and Schaelte G: Postoperative cognitive dysfunction: Incidence and prophylaxis. Anaesthesist 59: 177-184; quiz 185, 2010.

2. Deiner S and Silverstein JH: Postoperative delirium and cognitive dysfunction. Br J Anaesth 103 Suppl 1: i41-i46, 2009.

3. Seitz DP, Shah PS, Herrmann N, Beyene J and Siddiqui N: Exposure to general anesthesia and risk of Alzheimer's disease: a systematic review and meta-analysis. BMC Geriatr 11: 83, 2011.

4. Hu Z, Ou Y, Duan K and Jiang X: Inflammation: a bridge between postoperative cognitive dysfunction and Alzheimer's disease. Med Hypotheses 74: 722-724, 2010.

5. Lin D, Cao L, Wang Z, Li J, Washington JM and Zuo Z: Lidocaine attenuates cognitive impairment after isoflurane anesthesia in old rats. Behav Brain Res 228: 319-327, 2012.

6. Cohendy R, Brougere A and Cuvillon P: Anaesthesia in the older patient. Curr Opin Clin Nutr Metab Care 8: 17-21, 2005.

7. RosczykHA,Sparkman NL and Johnson RW: Neuroinflammation and cognitive function in aged mice following minor surgery. Exp Gerontol 43: 840-846, 2008.
8. Teeling JL and Perry VH: Systemic infection and inflammation in acute CNS injury and chronic neurodegeneration: underlying mechanisms. Neuroscience 158: 1062-1073, 2009.

9. Glezer I, Simard AR and Rivest S: Neuroprotective role of the innate immune system by microglia. Neuroscience 147: 867-883, 2007.

10. Crack PJ and Bray PJ: Toll-like receptors in the brain and their potential roles in neuropathology. Immunol Cell Biol 85: 476-480, 2007

11. Okun E, Griffioen KJ, Lathia JD, Tang SC, Mattson MP and Arumugam TV: Toll-like receptors in neurodegeneration. Brain Res Rev 59: 278-292, 2009.

12. Haseneder R, Kochs E and Jungwirth B: Postoperative cognitive dysfunction. Possible neuronal mechanisms and practical consequences for clinical routine. Anaesthesist 61: 437-443, 2012 (In German).

13. Shao J, Liu T, Xie QR, et al: Adjudin attenuates lipopolysaccharide (LPS)- and ischemia-induced microglial activation. J Neuroimmunol 254: 83-90, 2012.

14. Kamer AR, Galoyan SM, Haile M, et al: Meloxicam improves object recognition memory and modulates glial activation after splenectomy in mice. Eur J Anaesthesiol 29: 332-337, 2012.

15. Cai ZY, Yan Y and Chen R: Minocycline reduces astrocytic reactivation and neuroinflammation in the hippocampus of a vascular cognitive impairment rat model. Neurosci Bull 26: 28-36, 2010.

16. Moller JT, Cluitmans P, Rasmussen LS, et al: Long-term postoperative cognitive dysfunction in the elderly ISPOCD1 study. ISPOCD investigators. International Study of Post-Operative Cognitive Dysfunction. Lancet 351: 857-861, 1998.

17. Zhang Z, Zhang ZY, Wu Y and Schluesener HJ: Immunolocalization of Toll-like receptors 2 and 4 as well as their endogenous ligand, heat shock protein 70 , in rat traumatic brain injury. Neuroimmunomodulation 19: 10-19, 2012.

18. Krynetskaia NF, Phadke MS, Jadhav SH and Krynetskiy EY: Chromatin-associated proteins HMGB1/2 and PDIA3 trigger cellular response to chemotherapy-induced DNA damage. Mol Cancer Ther 8: 864-872, 2009.

19. Zheng Z, Yuan R, Song M, et al: The toll-like receptor 4-mediated signaling pathway is activated following optic nerve injury in mice. Brain Res: Oct 24, 2012 (Epub ahead of print). doi: 10.1016/j.brainres.2012.10.014.

20. Brown J, Wang H, Hajishengallis GN and Martin M: TLR-signaling networks: an integration of adaptor molecules, kinases, and cross-talk. J Dent Res 90: 417-427, 2011.

21. Li H Xu H and Sun B: Lipopolysaccharide regulates MMP-9 expression through TLR4/NF- $\kappa \mathrm{B}$ signaling in human arterial smooth muscle cells. Mol Med Report 6: 774-778, 2012.

22. Perry VH, Nicoll JA and Holmes C: Microglia in neurodegenerative disease. Nat Rev Neurol 6: 193-201, 2010.

23. Song M, Jin J, Lim JE, et al: TLR4 mutation reduces microglial activation, increases Abeta deposits and exacerbates cognitive deficits in a mouse model of Alzheimer's disease. J Neuroinflammation 8: 92, 2011.

24. Hua F, Ma J, Ha T, et al: Activation of Toll-like receptor 4 signaling contributes to hippocampal neuronal death following global cerebral ischemia/reperfusion. J Neuroimmunol 190: 101-111, 2007.

25. Pascual M, Balino P, Alfonso-Loeches S, Aragon CM and Guerri C: Impact of TLR4 on behavioral and cognitive dysfunctions associated with alcohol-induced neuroinflammatory damage. Brain Behav Immun 25 Suppl 1: S80-S91, 2011.

26. Xie Z and Tanzi RE: Alzheimer's disease and post-operative cognitive dysfunction. Exp Gerontol 41: 346-359, 2006.

27. Hartholt KA, van der Cammen TJ and Klimek M: Postoperative cognitive dysfunction in geriatric patients. Z Gerontol Geriatr 45: 411-416, 2012. 